\title{
Current strategies to circumvent the antiviral immunity to optimize cancer virotherapy
}

\author{
Dong Ho Shin, ${ }^{1}$ Teresa Nguyen, ${ }^{1}$ Bulent Ozpolat, ${ }^{2}$ Frederick Lang, ${ }^{3}$ Marta Alonso, ${ }^{4}$ \\ Candelaria Gomez-Manzano, ${ }^{1}$ Juan Fueyo ${ }^{1}$
}

To cite: Shin DH, Nguyen T, Ozpolat B, et al. Current strategies to circumvent the antiviral immunity to optimize cancer virotherapy. Journal for ImmunoTherapy of Cancer 2021;9:e002086. doi:10.1136/ jitc-2020-002086

Accepted 02 March 2021
Check for updates

(C) Author(s) (or their employer(s)) 2021. Re-use permitted under CC BY-NC. No commercial re-use. See rights and permissions. Published by BMJ.

${ }^{1}$ Department of Neuro-Oncology, The University of Texas MD Anderson Cancer Center, Houston, Texas, USA ${ }^{2}$ Department of Experimental Therapeutics, University of Texas MD Anderson Cancer Center, Houston, TX, USA

${ }^{3}$ Department of Neurosurgery, The University of Texas MD Anderson Cancer Center, Houston, TX, USA

${ }^{4}$ Department of Pediatrics, Clinica Universidad de Navarra, Pamplona, Navarra, Spain

Correspondence to

Dr Juan Fueyo;

jfueyo@mdanderson.org

\section{ABSTRACT}

Cancer virotherapy is a paradigm-shifting treatment modality based on virus-mediated oncolysis and subsequent antitumor immune responses. Clinical trials of currently available virotherapies showed that robust antitumor immunity characterizes the remarkable and long-term responses observed in a subset of patients. These data suggest that future therapies should incorporate strategies to maximize the immunotherapeutic potential of oncolytic viruses. In this review, we highlight the recent evidence that the antiviral immunity of the patients may limit the immunotherapeutic potential of oncolytic viruses and summarize the most relevant approaches to strategically redirect the immune response away from the viruses and toward tumors to heighten the clinical impact of viro-immunotherapy platforms.

\section{INTRODUCTION}

The immune system is exceptionally equipped to identify and destroy pathogens, and when successfully directed against cancer-derived antigens, can elicit powerful anticancer effects. Accordingly, the discovery that bacterial infection led to tumor regression in some sarcoma patients laid the foundation for cancer immunotherapy. ${ }^{1} \mathrm{~A}$ similar principle underlies the use of tumor-selective viruses today. In response to viral infections, the intracellular interferon (IFN) response triggers innate immune responses, including activation of natural killer (NK) cells and phagocytes. Viral antigens transported to the lymph nodes by antigen presenting cells elicit robust adaptive cellular immune responses, activating virus-specific naïve and memory B-cells and T-cells. ${ }^{23}$ Activated B-cells secrete antibodies that interfere with the viral life cycle, including internalization into target cells and intracellular translocation of viral proteins. ${ }^{4}$ T-cells exert cytotoxic effects on virus infected cells. Thus, the broad target spectrum of the innate immunity steers the deployment of a shaped, virus-specific adaptive immune response for specific and longterm protection against viral infections.
Two recently published phase 1 clinical trials demonstrated how infection of gliomas with replication-competent adenoviruses $(\mathrm{AdV})$ or recombinant polioviruses triggers immune responses that rapidly eradicate the therapeutic viruses in most patients, yet induce remarkable and longlasting anti-tumor effects in just $20 \%$ of the patients. ${ }^{56}$ A phase III clinical trial of the oncolytic herpes simplex virus (HSV) talimogene laherparepvec (T-Vec) for the treatment of advanced melanoma also demonstrated a $16.3 \%$ durable response rate and a $33 \%$ 5 -year survival rate, ${ }^{7}$ whereas this regimen has efficiently seroconverted all patients within 3-4 weeks, suggesting a successful induction of antiviral immunity. ${ }^{8}$

Therefore, a distinct discrepancy exists between the prevalence of anti-viral immunity and the dearth of the subsequent antitumorous immunity that can be attributed to many factors. Virotherapy is now accepted as a form of immunotherapy, ${ }^{9}$ and despite the fact that oncolytic viruses (OVs) have been shown to reverse immunosuppressive tumor microenvironments, factors that limit other conventional immunotherapies, such as T-cell exhaustion, may also be of relevance to virotherapy. ${ }^{10}$ To overcome these limitations, combination therapies of immune checkpoint inhibitors with cancer vaccines or other immune modulators have been explored, and multiple clinical trials are currently testing the effect of combining OVs with immune checkpoint inhibitors. ${ }^{11}$ In addition, OVs can face different challenges from other forms of immunotherapies in that viruses are highly immunogenic and, therefore, are subject to rapid destruction by the immune system. Since virotherapy's clinical efficacy can depend on sufficient initial virus replication, novel strategies to restrict the immune destruction of viruses and redirect the dominant immune response towards 
cancer cells may be necessary. In this review, we discuss how engineering less immunogenic and "stealth" OVs or developing selective immune tolerance for viral antigens may enhance the anticancer effect of virotherapies.

\section{SHIELDING OVS FROM THE INNATE AND ADAPTIVE HUMORAL IMMUNITY}

While several studies have shown the importance of the immune system for long-lasting tumor control using OVs, preclinical and early-stage clinical trials have also suggested reduced virus replication and earlier clearance in immunocompetent hosts. ${ }^{12}$ As some OVs are administered systemically to treat inoperable tumors or disseminated metastases, the rapid neutralization of viruses by the humoral immunity including the complement system and antibodies represents a considerable obstacle for virotherapies. Complement proteins and antibodies facilitate the destruction of infected cells by interacting with effector cells or inhibiting cellular receptor binding, endosomal escape, nuclear transport of viral genomes and enveloped virion budding from host cells. ${ }^{413}$ Furthermore, OVs derived from prevalent viruses like AdV or HSV face more significant risks of neutralization by predeveloped antibodies. ${ }^{14} 15$ This section discusses three main strategies to avoid the hindrance by the humoral immunity: protective coatings, cellular vehicles, and viral genome modifications, as shown in figure 1 . We then examine the alternate view that humoral immunity may be beneficial for OV efficacy. These strategies mitigate both antibodymediated and complement-mediated neutralization. ${ }^{16}$

\section{Coatings}

Protective coatings are a straightforward approach to physically shield OVs and is one of the most intuitive strategies to counteract soluble immune factors. Various types have been explored, including chemical polymers, liposomes and cell-derived nanovesicles. ${ }^{17-19}$ These coatings can protect OVs from both passive and active immunity in immunodeficient and immunocompetent hosts. Graphene oxide protected measles virus (MV) from neutralizing antibodies and enhanced oncolysis, offering a survival advantage in subcutaneous HeLa-bearing mice with passive immunity through antiserum injection. ${ }^{17}$ Layer-by-layer deposition of ionic polymers also protected MV from antibodies and showed improved virus activity and tumor control in immunized, immunocompetent mice with subcutaneous lung carcinoma. ${ }^{20}$ Coatings not only protect OVs from preexisting antibodies but also can limit the production of new antibodies. Liposomes used to encapsulate the Getah-like alphavirus M1 or the plasmid DNA of AdV protected OVs from antibodies in vitro and reduced the production of neutralizing antibodies in mice after systemic virus administration. ${ }^{18} 21$ Systemic delivery of OVs can also be limited by untargeted dilution of the effective virus dose in the system. Coatings can be engineered to incorporate tumor-targeting ligands that facilitate OV trafficking to tumors. In this regard, bioengineered cell membrane nanovesicles embedded with preS1 ligand significantly enhanced the delivery of AdV to tumors expressing the preS1-specific receptor sodium taurocholate cotransporting polypeptide, suppressed the secretion of inflammatory cytokines interleukin 6 (IL-6) and tumor necrosis factor (TNF)- $\alpha$ and protected the virus from neutralizing antibodies. ${ }^{19}$ Caution is required with this approach, however, as physical barriers to encapsulate OVs may hinder the interaction between viruses and tumor cell receptors to reduce infectivity. ${ }^{22}$ In addition, potential limitations for manufacturing coated viruses include potentially high costs, limitations for large-scale production, and stability during storage. $^{23}$

\section{Cellular carriers}

Cells loaded with OVs ex vivo and injected back into patients offer a different approach to protect OVs. Cellular carriers derived from endothelial cells, T-cells, mesenchymal stromal cells, tumor cells as well as mesenchymal, neuronal, menstrual blood-derived and adiposederived stem cells have been explored to deliver vaccinia virus (VV), vesicular stomatitis virus (VSV), MV, HSV, $\mathrm{AdV}$ and reovirus. ${ }^{24-32}$ Carrier cells derived from solid tumors can be easily infected with OVs but a safety concern for injecting cancer cells into patients may limit further clinical development. ${ }^{24}$ Due to their loading capability, immune evading phenotypes, and intrinsic tumor tropism, mesenchymal or neuronal stem cells are attractive candidates as cell vehicles. In brain tumors, presence of the blood-brain barrier and the influx of blood and cerebrospinal fluid following tumor resection impede successful virus delivery during systemic and intratumoral virus injections. Cellular carriers can provide a solution to such delivery problems. Oncolytic AdV ICOVIR17 loaded onto mesenchymal stem cells (MSCs) with tumor tropism showed significantly enhanced viral delivery to brain tumors, reduced tumor growth and increased animal survival. ${ }^{33}$ Intracarotid injection of oncolytic HSV loaded onto MSCs, but not the virus alone, tracked metastatic melanoma lesions in the brain, and prolonged the survival of mice. ${ }^{27}$ High costs associated with loading cells with OVs ex vivo may be reduced using off-the-shelf, allogeneic stem cells. ${ }^{30}$ Delivery of OVs using cellular carriers is already being investigated in the clinical arena. ${ }^{34}$ For instance, MSCs loaded with AdV were shown to be safe in pediatric and adult solid tumors. ${ }^{35}$ A phase I/II study of an oncolytic MV delivered with MSCs to ovarian cancer is ongoing at Mayo Clinic (NCT02068794). Another phase I trial at MD Anderson Cancer Center examines the delivery of our adenoviral construct Delta-24-RGD to gliomas using human MSCs (NCT03896568). Neural stem cells are used to deliver oncolytic AdV to malignant gliomas at the Northwestern University (NCT03072134). T-cells are another attractive candidate carrier for OVs. Compared with stem cells that home to stromal regions, T-cells deliver OVs to tumors through direct contact. ${ }^{26}$ Chimeric antigen receptor (CAR) T-cells can also be used 
Overcoming T-cell Immunodominance

Shielding OV from Humor Immmunity

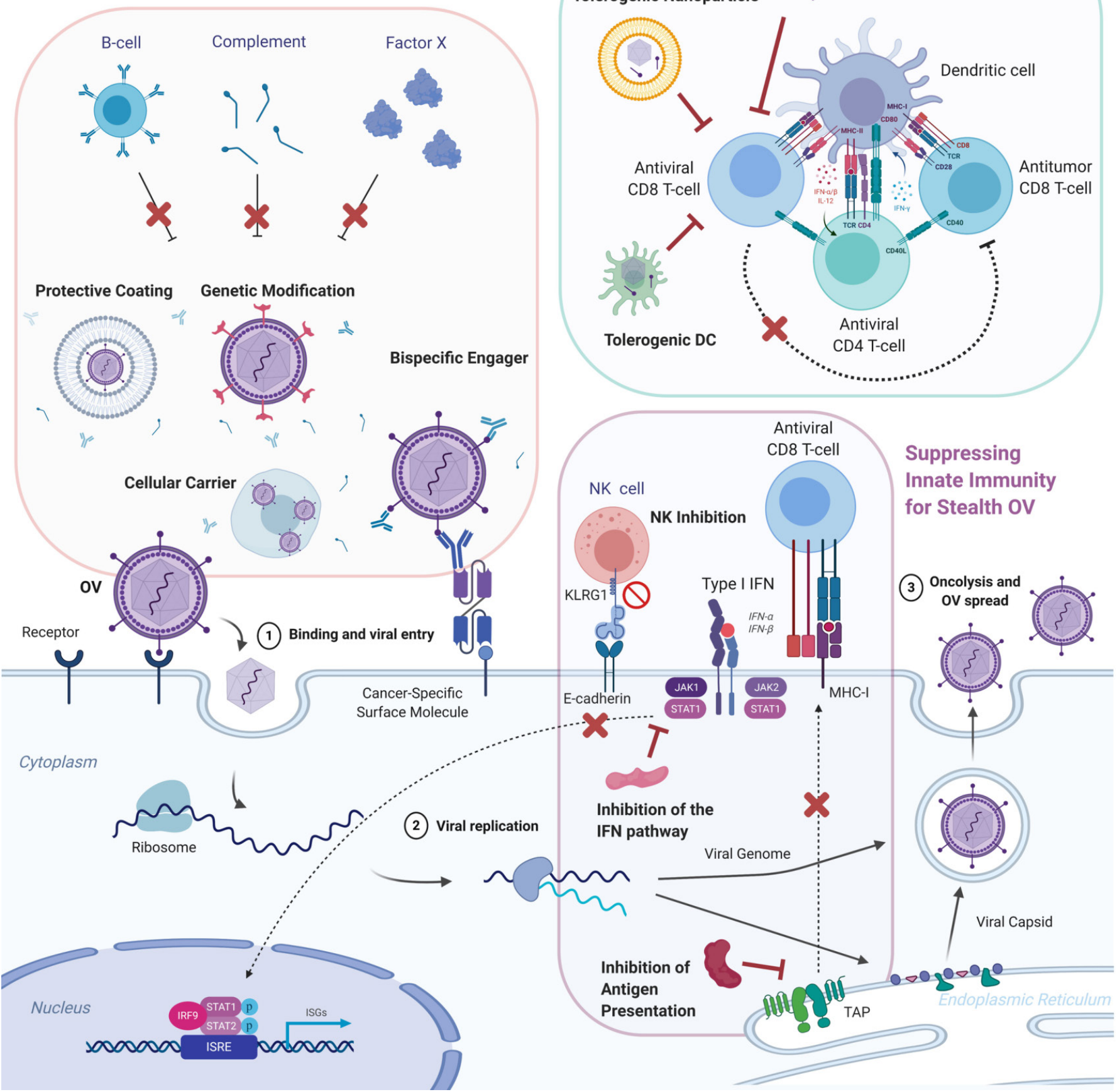

Figure 1 Methods to circumvent antiviral immunity. Oncolytic viruses (OVs) exert their effect by infecting the cancer cells, replicating, and inducing oncolysis. limmunogenic cell death of the tumor subsequently activates anti-viral and anti-tumor T cells. However, viral activity is limited by the humoral, innate and adaptive cellular immune components of the host. Several approaches aim to counteract each arm of the immune system to protect the viruses and maximize tumor regression. OVs can be shielded from the humoral immune responses of B cells and the complement pathway through encapsulation OVs in protective coatings and cellular carriers, genetic modification of the viral capsid to reduce expression of common viral epitopes, and administration of bispecific engagers that can bind neutralizing antibodies and tumors cells on different ends. To confer OVs stealth characteristic, the innate immunity can be tamed through the inhibition of the anti-viral interferon (IFN) pathway, the natural killer cells and antigen presentation. T cell immunodominance from viral antigens can be overcome through nanoparticle-enveloped viral antigens and tolerogenic dendritic cells. ISG, interferon-stimulated gene; ISRE, interferonstimulated response element; MHC, major histocompatibility complex; NK, natural killer; TAP, transporter associated with antigen processing. 
as vehicles to transport the OVs to the tumors. Oncolytic VSV or recombinant VV could be loaded onto murine T-cells or human HER2-CAR T-cells without impacting CAR expression, T-cell viability or functionality. ${ }^{36}$

\section{Genetic modifications of OVs}

Most neutralizing antibodies target a limited number of epitopes on an antigen. Identifying and genetically modifying such epitopes on OVs could help evade preexisting antibodies from prior virus exposure. For AdV, the hypervariable regions (HVRs) of hexon are the dominant epitopes for B-cells in humans. ${ }^{37}$ Replacing HVRs negated the activity of serotype-specific antibodies. ${ }^{38}$ Directed clonal evolution can be used to select a clone with low seroprevalence and high oncolytic potency. Clonal expansion of oncolytic AdV d11520 produced the clone ColoAd 1 with a 10 -fold lower sera inhibition and a higher oncolytic ability. ${ }^{39} \mathrm{VV}$ produces a particular form of virions called extracellular enveloped virus (EEV) that are protected from neutralizing antibodies by the host-derived lipid bilayer. Introducing a point mutation in the A34R gene resulted in more EEV production that enhanced virus spread and production of progeny virions, protected the virus from neutralizing antibodies, and prolonged animals' survival. ${ }^{40}$ In a different approach, deglycosylation of VVs reduced TLR2-mediated pathogen recognition signaling and protected the virus from neutralizing antibodies. ${ }^{41}$ For HSV, replacement of residues 2-24 with antiepidermal growth factor receptor (EGFR) single-chain variable fragment $(\mathrm{scFv})$ and point mutations P54Q and T213M in the viral glycoprotein D decreased the binding of neutralizing antibodies. ${ }^{42}$

\section{The alternate view: beneficial roles of antibodies}

Although neutralizing antibodies are generally detrimental for virus replication, some recent evidence suggests that they may not play a decisive role at sufficiently high doses of virus. Systemic injection of AdV, Newcastle disease virus (NDV), HSV, VV and reovirus in clinical trials have demonstrated virus delivery to tumors and induction of antitumor immunity, although to a limited degree. ${ }^{43}$ Importantly, a window-of-opportunity clinical trial prior to resection of colorectal cancer metastases in the liver showed that intravenously injected oncolytic reovirus was transported to tumors despite the presence of neutralizing antibodies. ${ }^{44}$ Further investigation revealed that CD11b+ cells facilitated the uptake of the reovirus-antibody complex, and expansion of such cell population using Granulocyte-macrophage colony-stimulating factor (GMCSF) augmented the therapy in mice with melanoma, but only in the presence of neutralizing antibodies. ${ }^{25}$ In a different strategy, pre-existing neutralizing antibodies can be exploited through bispecific engagers that bind to neutralizing antibodies at one end and to tumor cells at the other end. Bispecific engagers retargeted antibodybound AdV to tumors, attracted activated NK cells and CD8+ T cells to tumors, and improved the survival of mice with subcutaneous polysialic acid expressing tumors. ${ }^{45}$
Bispecific engagers can also be used to ensure safety associated with nonspecific viral infection and augment tissue-specific delivery. Bispecific fusion protein that targeted the viral hemagglutinin-neuraminidase protein of the NDV, on one end, and the IL 2 receptor, on the other end, blocked the native cell binding and retargeted the virus to IL-2R positive lymphoma cells both in vitro and in vivo. ${ }^{46}$ Bispecific T-cell engagers also increase T-cell interaction with OV infected tumors and are rapidly moving to clinical trials as reviewed by Slaney and collages. ${ }^{48}$ Interestingly, some studies report that pre-vaccination can augment the efficacy of virotherapy. NDV-immunized mice showed more effective clearance of subcutaneous melanoma when compared with naive mice. ${ }^{49}$ However, immunization led to decreased virus replication, suggesting that the preexisting antibodies limit oncolysis. The observed therapeutic benefit was dependent on increased activities of NK cells and CD8+ Tcells, indicating that the humoral immune response is still a hurdle that should be overcome.

\section{Section summary}

In summary, cumulative evidence suggests that protecting OVs from the host's humoral immune response may improve the persistence of virus in the system and increase the chances for the development of a robust antitumor immune response. Some studies suggest otherwise that immune responses against the virus enhance their anticancer efficacy. Although there is evidence to support both sides of the argument, it seems clear that most of the current literature agrees that inhibition of the humoral immunity is essential for successful viral replication if the therapeutic virus should be administered systemically.

\section{THE DOUBLE-EDGED EFFECT OF SUPPRESSING THE INNATE IMMUNITY}

The innate immune system recognizes and provides a quick response to a diverse array of foreign antigens. Pattern recognition receptors activated by viral DNAs and RNAs recruit adaptor proteins and phosphorylate transcription factors to induce the type I IFN response. ${ }^{50}$ RIGI-like RNA helicases also bind to viral RNAs and activate mitochondrial antiviral signaling, which exhibits a prionlike conformational switch to activate and propagate the antiviral signaling cascade. ${ }^{51}$ Expression of IFN-related genes halts viral replication, activates cytokine production, and recruits NK cells to kill infected cells. ${ }^{52}$ The innate immune system also steers the activation of the adaptive immune response by orchestrating the presentation of viral and tumorous antigens. Since activation of the adaptive immune response against tumor-derived antigens is critical for OV efficacy, the specific roles of each component of the innate immunity in relevance to tumor therapy is often debated. This section discusses how the intracellular IFN pathway, NK cells and the antigen presentation pathway affect virotherapy. 


\section{IFN pathway}

Many cancer cells have defects in the type I IFN pathway that make them more permissive to $\mathrm{OV}$ replication compared with noncancerous cells. ${ }^{50}$ Furthermore, IFN- $\alpha$ can antagonize OV by reducing replication and blocking virus-mediated apoptosis. ${ }^{53}$ Accordingly, treatment of cancer cells with inhibitors of the IFN response, such as the IKK-2 inhibitor, TPCA-1 or the JAK1/2 inhibitor, ruxolitinib, augmented the replication of respiratory syncytial, influenza, measles and mumps viruses. ${ }^{54}$ Ruxolitinib treatment, in conjunction with oncolytic HSV significantly enhanced CD8+ T cell activation in the tumor microenvironment and prolonged the survival of mice with malignant peripheral nerve sheath tumors. ${ }^{55}$ A clinical trial at Mayo Clinic is currently testing the effect of combining oncolytic VSV expressing IFN $\beta$ with or without ruxolitinib for patients with stage IV or recurrent endometrial cancer (NCT03120624). Due to neurotoxicity of VSV observed in some mice, IFN $\beta$ was incorporated into the viral genome to suppress viral replication in non-cancerous tissues. Ruxolitinib resensitized cancer cells that are resistant to VSV-IFN $\beta{ }^{56}$ Numerous studies are testing the effects of other small molecules that converge on the IFN pathway as reviewed by Phan et $a l^{57}$ IFN-binding decoy receptors also augmented oncolysis, improved survival and decreased the number of colorectal carcinoma metastases in mice treated with oncolytic rhabdoviruses. ${ }^{58}$ While reports of enhanced virus efficacy on inhibition of the IFN function are promising, measures to ensure safety should be considered. For example, arming the Semliki Forest virus with the VV-encoded type I IFN decoy receptor B18R resulted in neurotoxicity in vivo due to uncontrolled viral replication in healthy brains. ${ }^{59}$ Further investigation is required to clarify which antagonists of the IFN pathway show the broadest therapeutic index. Chemical compounds also offer solutions to the inhibitory effect of the IFN pathway. Vanadium compounds suppressed the antiviral type I IFN response while promoting the proinflammatory type II IFN response, and enhanced the replication of RNA viruses like VSV, measles and sindbis virus, increasing their antitumor effects. ${ }^{60}$ Dimethyl fumarate and related fumaric acid esters also inhibited the type I IFN response and in combination with oncolytic VSV led to better survival of tumor-bearing mice. ${ }^{61}$

\section{NK cells}

The role of NK cells during OV therapy seems to be highly context-dependent. ${ }^{62}$ On the one hand, NK cells reinforce OV therapy by exerting their cytotoxic effect on virus-infected cancer cells. Consistently, enhancement of NK activity or trafficking through OV-induced transgenes or pharmacological treatments resulted in greater tumor regression. ${ }^{63-65}$ Depletion of NK cells or inhibition of NK perforin activity during oncolytic HSV therapy reduced tumor lysis. ${ }^{66}$ NK depletion also abolished the antimelanoma effect of an oncolytic Maraba virus treatment. ${ }^{65}$ These studies support the role of NK cells in augmenting
OV-mediated tumor lysis. On the other hand, NK cells interfere with viral replication and may offset the effect of OVs. NK cell depletion nullified the expression of inflammatory genes responsible for rapid clearance of oncolytic HSV, and improved the survival of mice with glioblastoma xenografts and syngeneic tumors. ${ }^{67}$ Furthermore, NK natural cytotoxicity receptor knock-out $\left(\mathrm{Ncrl}^{--}\right)$mice exhibited better response to HSV therapy ${ }^{67}$ In a similar approach, OVs can be engineered to express transgenes including E-cadherin, which binds to NK inhibitory receptor, or UL141 cytomegalovirus protein, which downregulates the NK activation signal. ${ }^{6869}$ These engineered OVs suppressed NK activity and tumor infiltration, potentiated viral spread, and prolonged the survival of animals with glioblastoma or hepatocellular carcinoma.

The contradicting results highlight the NK cells' complex role in OV therapy that may depend on the treatment kinetics and the activation of specific subpopulations of NK cells. Suppression of NK cells during the initial phase of virus replication, followed by activation of NK cells to elicit adaptive immune responses, can maximize virotherapy's efficacy. ${ }^{62}$ Thus, transient depletion of endogenous NK cells prior to oncolytic HSV treatment followed by exogenous NK cell injection post virus treatment yielded the maximum efficacy in mice with patientderived primary glioblastoma. ${ }^{70}$

The recent development of chimeric antigen receptor engineered NK (CAR-NK) cells may offer a new opportunity to unleash the full potential of NK-mediated tumor clearance without compromising virus replication. NK cell activity can be redirected from virus-infected cells towards cancer cells by engineering the NK receptors. In this regard, the combination of HSV with NK cells expressing EGFR-CAR significantly enhanced the therapeutic efficacy compared with either monotherapy in a murine model of EGFR+ breast cancer brain metastasis. ${ }^{71}$ Virotherapy enhances NK cell infiltration into tumors and may aid the homing of CAR-NK to tumors.

\section{Antigen presentation pathway}

Antigen presentation can be modulated through inhibition of the transporter associated with antigen processing (TAP). HSV-mediated expression of a TAP inhibiting protein, bovine herpesvirus UL49.5, reduced Major histocompatibility complex (MHC) expression on cell surfaces, diminished recognition of infected cells by immune cells, prolonged viral persistence and improved survival of mice bearing subcutaneous bladder or breast cancers. $^{72}$

\section{Section summary}

The strategies described in this section address the complexity of the interactions between OVs and different components of the innate immunity. Despite some reports of unwanted toxicity during attempts to manipulate the IFN pathway, many proposed strategies, including small molecule inhibitors, resulted in improved efficacy without compromised safety. Furthermore, more studies 
are required to clarify whether contradictions in the literature are based on the contextual roles of NK cells or the timing of their activation during OV treatment. New insights will be helpful in determining when the innate immunity should be manipulated in clinical trials.

\section{METHODS TO SUBVERT T-CELL IMMUNODOMINANCE BY VIRAL ANTIGENS}

Preclinical and clinical evidence supports the critical role of T-cells in tumor regression after OV infection. ${ }^{11} \mathrm{OV}$ efficacy is suppressed in immunodeficient mice and upon CD8+ Tcell depletion. ${ }^{73}$ Furthermore, arming OVs with T-cell activators such as OX40L or GITRL magnifies their antitumor effects. ${ }^{74}$ Consistently, the combination of OVs and immune checkpoint inhibitors yield improved results compared with either monotherapy. ${ }^{11} 7677$ While many clinical trial results are pending, a phase $1 \mathrm{~b}$ trial testing talimogene laherparepvec (T-Vec) combined with the anti-PD-1 antibody, pembrolizumab, showed favorable changes in the tumor microenvironment, resulting in a $62 \%$ overall response rate and a $33 \%$ complete response rate in metastatic melanoma. ${ }^{78}$ Results from a phase I/ II clinical trial (NCT02798406) testing the combination of our Delta-24-RGD and anti-PD1 antibody, pembrolizumab, are encouraging with a 6 -month overall survival of $91 \%$ and $47 \%$ of the patients showing clinical benefits. ${ }^{79}$

Despite the undeniable contribution of T-cells for virotherapy, one overlooked aspect of virus-induced antitumor immunity is the distinction between the viral antigen-specific T-cells and the tumor antigen-specific T-cells. Viruses are quickly eliminated by the immune system after injection, and the tumor bulk that has not been virus infected is slowly destroyed by the tumorspecific immune cells. Ultimately, T-cells against tumor antigens are responsible for complete eradication and long-term protection from tumors. Many of the current strategies indiscriminately boost T-cells that target tumor and viral antigens. Still, evidence suggests that an overwhelming immune response against the highly immunogenic viral antigens may outcompete and suppress the subdominant tumor-specific T-cell response due to immunodominance. ${ }^{80}$ Vaccination with tumor-associated antigens is a viable approach to boost anti-tumor immunity that has been explored, for example, with oncolytic Maraba virus. ${ }^{81}$ However, this approach requires a priori knowledge of tumor antigens for each patient or cancer type. As such, strategies to restrict the anti-viral immune response will be critical to restore the balance of immune responses and enhance the antitumor effect of $\mathrm{OV}$ therapy.

As noted, immune suppression during the initial phases of OV infection may enhance viral replication and support tumor clearance. However, a non-specific immune suppression is not ideal because it prevents the activation of tumor-specific T-cells. Antigen-specific immune tolerance has not been well explored in the context of OV therapy, but studies on autoimmunity and viral vectors for gene therapy provide deep insights into this crucial angle of virotherapy. This section discusses three strategies: nanoparticle delivery of viral antigens, tolerogenic dendritic cells (DCs) and CAR T-cells, as shown in figure 1.

\section{Nanoparticle delivery of viral antigens}

Nanoparticles typically range between 10 and $100 \mathrm{~nm}$ and can be used to deliver payloads such as cancer therapeutics, proteins, peptides, oligonucleotides and imaging agents. An ideal nanocarrier should be safe, non-immunogenic, biocompatible, biodegradable, bypass rapid hepatic or renal clearance and should preferentially target preferred tissues or tumors for maximal efficacy. Nanoparticles made of biodegradable nanomaterials vary from natural or synthetic to polymers, carbon nanotubes, metal-based and silica-based nanoparticles. Each of the nanoparticle system has its own unique tissue distribution, cell uptake and toxicity based on their physicochemical features, including charge, size, shape and hydrophobicity.

Liposomal nanoparticles are a popular nanocarrier system that have been extensively employed to enhance the efficiency of drug delivery to tumors due to their high degree of biocompatibility during systemic administration. ${ }^{82}$ Positively charged or cationic liposomes are highly effective in capturing negatively charged molecules such as oligonucleotides, and negatively charged liposomes are rapidly cleared from circulation and captured by macrophages. Neutral nanoliposomes composed of dioleoyl phosphatidyl-choline provide robust delivery of the payload following systemic administration of peptides and oligonucleotides. ${ }^{83-85}$ The net surface charges (negative, positive or neutral) play important roles in their adjuvant activity when admixed with protein antigens.

Systemic delivery of antigens using nanoparticles can closely mimic apoptotic bodies that are cleared to induce immunological tolerance. During the natural process of apoptotic cell clearance, 'eat-me' signals such as the localization of phosphatidylserine (PS) on the cell surface triggers the uptake of apoptotic cells by professional phagocytes such as macrophages and immature DCs. Antigen presenting cells use multiple receptors, including members of Tyro-3, Axl, and MER (TAM) receptor kinases, Stabilin-2, CD36 and CD68 to recognize the 'eat me' signals. Phagocytosis of the apoptotic cells promotes the release of anti-inflammatory signals and presentation of autoantigens in a tolerogenic fashion by DCs. Membrane-associated ligands on apoptotic cells interact with receptors on DCs to disrupt TLR signaling and block DC maturation. ${ }^{86}$ Other phagocytic pathways independent of PS, including integrin-based systems or scavenger receptors, also mediate apoptotic cell clearance. ${ }^{87}$

Studies indicate that uptake of liposomes by macrophages increased production of transforming growth factor- $\beta,{ }^{88}$ consistent with the cytokine profile of immunosuppressive M2 type of macrophages. ${ }^{89}$ Furthermore, copresentation of antigen and ligands of B-cell sialic 
acid binding immunoglobulin-like lectins on a liposomal nanoparticle induced antigen-specific tolerance. ${ }^{90}$ Lipid based liposomal nanoparticles expressing PS mimicked apoptotic bodies and induced antigen-specific immune tolerance ${ }^{91}$ Biodegradable polymers like poly(lactic-coglycolic acid) were recognized by scavenger receptors MARCO, resulting in uptake and presentation of antigens to induce immune tolerance. ${ }^{92}$ To enhance the efficiency of tolerance induction, nanoparticles can be loaded with immunosuppressive molecules, such as IL-10 or 2-(1'H-indole-3'-carbonyl)-thiazole-4-carboxylic acid methyl ester (ITE). ${ }^{93} 94$ In the context of virus-specific immune tolerance, synthetic particles encapsulating rapamycin synthetic vaccine particles carrying rapamycin (SVP-Rapa) when coadministered with adeno-associated virus vectors prevented the induction of anticapsid humoral and cell-mediated responses, caused tolerance, enhanced transgene transduction and allowed for repeated vector administration. ${ }^{95}$

\section{Tolerogenic DCs}

As described previously, antigen presenting cells play a central role in inducing immunological tolerance. Tolerogenic DCs can be generated through various methods independently of nanoparticles and can suppress T-cells through different mechanisms. A direct method to induce antigenspecific tolerance involves extracting DCs with tolerogenic properties and exposing them to antigens of interest ex vivo, followed by their reinfusion. Immunosuppressive corticosteroids combined with DC maturation cytokines TNF- $\alpha$, IL-1 $\beta$, IL-6 and PGE2 generated tolerogenic DCs in vitro. ${ }^{96}$ Tolerogenic DCs were also generated through internalization of immunoglobulin-coated AdV leading to pyroptosis and secretion of cytokines that promoted the semimaturation of bystander DCs. ${ }^{97}$ Incomplete maturation of DCs induced immune-suppressive regulatory T-cells and increased viral persistence. ${ }^{97}$ Interestingly, vaccination during the early stages of immune development can also induce tolerance through tolerogenic DCs. Subcutaneous injection of VSV in newborn mice could show, for the first time, that OV-specific tolerance was induced, which resulted in improved viral persistence and enhanced OV efficacy. ${ }^{98}$ Despite the intrinsic limitations of this neonatal approach for human application, development of tolerogenic vaccines against common $\mathrm{OV}$ antigens using immunosuppressants is an interesting possibility. Overall, the use of tolerogenic DCs is interesting from a therapeutic perspective because it would bypass the need for a non-specific and potentially dangerous immunosuppression that would have a deleterious effect on OV therapy.

\section{CAR T-cells}

Combination therapy involving OVs and tumor-specific CAR T-cells is another strategy to redirect the immune response towards tumor antigens. OV infection should generate conductive conditions to attract ectopic T-cells to the tumor through secretion of cytokines, overcome immunosuppression, and have a global immune effect. ${ }^{99}$ Furthermore,
OVs can be engineered to facilitate CAR T-cell therapy. For example, Ad5 $\Delta 24$.RANTES.IL-15 secreted chemokine RANTES, also known as CCL5, that attracted GD2.CAR T-cells to the tumor, and cytokine IL-15 that prolonged the persistence of CAR T-cells. This resulted in increased survival in a neuroblastoma mouse model compared with either monotherapy. ${ }^{100}$

\section{Section summary}

Strategies aiming to make T-cells strong allies of the OVs have the potential to produce a vertical impact in the viroimmunotherapy field. The combination of T-vec with immune checkpoint inhibitors improved the results of T-vec in metastatic cancers of melanoma. However, in most viroimmnotherapy trials, the anti-viral CD8+ Tcell immune response probably interferes with the development of a robust antitumor immune response. To avoid this interference, and in an attempt to shift the antigen immunodominance from viruses to tumor antigens, investigators should explore methods to generate immune tolerance to virus epitopes. Additionally, DCs can be targeted directly to acquire the necessary tolerance to virus antigens. In a different approach, genetically engineered T-cells can be combined with the administration of the virus. In this case, the viral infection may generate a chemotactic tumor environment to attract CAR T-cells to the tumor. Furthermore, viruses expressing cancer antigens can be combined with CAR T-cells targeted to those antigens to decrease off-target effects and facilitate the migration of the ectopic T-cell population to the tumor.

\section{CONCLUSIONS}

The paradigm-shifting discovery of oncolytic virotherapy as a particularly effective form of immunotherapy has prompted a series of new concepts and theories that should improve cancer virotherapy. A plethora of new strategies will potentially augment the oncolytic and anti-tumor effects by modulating the host immune response. Approaches to shield viruses from humoral immunity include protective coating, cell vehicles and genetic modifications of the virus. We should remember that suppressing the innate immunity may result in a double-edged effect, as in the case of NK cells. In the modulation of the T-cell mediated immunity, it might be essential to facilitate the immune tolerance of virus antigens that can be achieved by systemic delivery of dominant antigens of the OV, infusing tolerogenic DCs and combining viruses with antitumor CAR T-cells.

In conclusion, strategies designed to redirect the immune system from the virus to the tumor may be of great relevance to improve the prognosis of patients treated with OVs. Any attempt should be made to generate an alliance between the therapeutic virus and the host's immune system to work together against the tumor. This new knowledge should propel new modalities of immunotherapy and virotherapy of primary and metastatic solid tumors. Now, more than ever, the pace of advance in these exciting scientific fields is accelerating. 


\section{Twitter Marta Alonso @pumorister}

Contributors DHS and JF contributed to the conception; DHS researched literature; all authors contributed to the writing, editing and review of the manuscript.

Funding This work was supported in part by NIH/NCI grants P50 CA127001, F31 CA228207, and R01 CA256006; the Cancer Prevention and Research Institute of Texas (RP170066); the Department of Defense (Team Science Award CA160525); the John and Rebekah Harper Fellowship; the Marnie Rose Foundation; the J.P. Harris Brain Tumor Research Fund and the Bradley Zankel Foundation.

Competing interests FL, JF and CG-M are reporting ownership interest (including patents). CG-M and JF are consultants and shareholders of DNATrix.

\section{Patient consent for publication Not required.}

Provenance and peer review Not commissioned; externally peer reviewed.

Open access This is an open access article distributed in accordance with the Creative Commons Attribution Non Commercial (CC BY-NC 4.0) license, which permits others to distribute, remix, adapt, build upon this work non-commercially, and license their derivative works on different terms, provided the original work is properly cited, appropriate credit is given, any changes made indicated, and the use is non-commercial. See http://creativecommons.org/licenses/by-nc/4.0/.

\section{REFERENCES}

1 Coley WB. The diagnosis and treatment of bone sarcoma. Glasgow Med J 1936;126:128-64.

2 Kim TS, Hufford MM, Sun J, et al. Antigen persistence and the control of local $\mathrm{T}$ cell memory by migrant respiratory dendritic cells after acute virus infection. J Exp Med 2010;207:1161-72.

3 Olson MR, McDermott DS, Varga SM. The initial draining lymph node primes the bulk of the CD8 T cell response and influences memory $T$ cell trafficking after a systemic viral infection. PLoS Pathog 2012;8:e1003054.

4 Murin CD, Wilson IA, Ward AB. Antibody responses to viral infections: a structural perspective across three different enveloped viruses. Nat Microbiol 2019;4:734-47.

5 Lang FF, Conrad C, Gomez-Manzano C, et al. Phase I study of DNX-2401 (Delta-24-RGD) oncolytic adenovirus: replication and immunotherapeutic effects in recurrent malignant glioma. J Clin Oncol 2018;36:1419-27.

6 Desjardins A, Gromeier M, Herndon JE, et al. Recurrent glioblastoma treated with recombinant poliovirus. $N$ Engl J Med 2018;379:150-61.

7 Andtbacka RHI, Kaufman HL, Collichio F, et al. Talimogene laherparepvec improves durable response rate in patients with advanced melanoma. J Clin Oncol 2015;33:2780-8.

$8 \mathrm{Hu}$ JCC, Coffin RS, Davis CJ, et al. A phase I study of OncoVEXGM-CSF, a second-generation oncolytic herpes simplex virus expressing granulocyte macrophage colony-stimulating factor Clin Cancer Res 2006;12:6737-47.

9 Tang J, Shalabi A, Hubbard-Lucey VM. Comprehensive analysis of the clinical immuno-oncology landscape. Ann Oncol 2018;29:84-91.

10 Sharma P, Hu-Lieskovan S, Wargo JA, et al. Primary, adaptive, and acquired resistance to cancer immunotherapy. Cell 2017;168:707-23.

11 Nguyen T, Avci NG, Shin DH, et al. Tune up in situ autovaccination against solid tumors with oncolytic viruses. Cancers 2018;10:1-19.

12 Filley AC, Dey M. Immune system, friend or foe of oncolytic virotherapy? Front Oncol 2017;7:1-8.

13 Smith JG, Cassany A, Gerace L, et al. Neutralizing antibody blocks adenovirus infection by arresting microtubule-dependent cytoplasmic transport. J Virol 2008;82:6492-500.

14 Cheng C, Gall JGD, Kong W-pui, et al. Mechanism of ad5 vaccine immunity and toxicity: fiber shaft targeting of dendritic cells. PLoS Pathog 2007;3:0239-45.

15 Bradley H, Markowitz LE, Gibson T, et al. Seroprevalence of herpes simplex virus types 1 and 2--United States, 1999-2010. J Infect Dis 2014;209:325-33.

16 Gentile CM, Borovjagin AV, Richter JR, et al. Genetic strategy to decrease complement activation with adenoviral therapies. PLOS One 2019;14:1-15

17 Xia M, Luo D, Dong J, et al. Graphene oxide arms oncolytic measles virus for improved effectiveness of cancer therapy. J Exp Clin Cancer Res 2019;38:1-16.
18 Wang $\mathrm{Y}$, Huang $\mathrm{H}$, Zou $\mathrm{H}$, et al. Liposome encapsulation of oncolytic virus $\mathrm{M} 1$ to reduce immunogenicity and immune clearance in vivo. Mol Pharm 2019;16:779-85.

19 Lv P, Liu X, Chen X, et al. Genetically engineered cell membrane Nanovesicles for oncolytic adenovirus delivery: a versatile platform for cancer virotherapy. Nano Lett 2019;19:2993-3001.

20 Nosaki K, Hamada K, Takashima Y, et al. A novel, polymercoated oncolytic measles virus overcomes immune suppression and induces robust antitumor activity. Mol Ther Oncolytics 2016;3:16022.

21 Aoyama K, Kuroda S, Morihiro T, et al. Liposome-encapsulated plasmid DNA of telomerase-specific oncolytic adenovirus with stealth effect on the immune system. Sci Rep 2017;7:1-10.

22 Francini N, Cochrane D, Illingworth S, et al. Polyvalent diazonium polymers provide efficient protection of oncolytic adenovirus Enadenotucirev from neutralizing antibodies while maintaining biological activity in vitro and in vivo. Bioconjug Chem 2019;30:1244-57.

23 Hill C, Carlisle R. Achieving systemic delivery of oncolytic viruses. Expert Opin Drug Deliv 2019;16:607-20.

24 Power AT, Wang J, Falls TJ, et al. Carrier cell-based delivery of an oncolytic virus circumvents antiviral immunity. Mol Ther 2007;15:123-30.

25 llett E, Kottke T, Donnelly O, et al. Cytokine conditioning enhances systemic delivery and therapy of an oncolytic virus. Mol Ther 2014;22:1851-63.

26 Onishi T, Tazawa H, Hashimoto $\mathrm{Y}$, et al. Tumor-specific delivery of biologics by a novel T-cell line HOZOT. Sci Rep 2016;6:1-10.

27 Du W, Seah I, Bougazzoul O, et al. Stem cell-released oncolytic herpes simplex virus has therapeutic efficacy in brain metastatic melanomas. Proc Natl Acad Sci U S A 2017;114:E6157-65.

28 Berkeley RA, Steele LP, Mulder AA, et al. Antibody-neutralized reovirus is effective in oncolytic virotherapy. Cancer Immunol Res 2018;6:1161-73.

29 Xu C, Xia M, Meng G, et al. Carrier cells for delivery of oncolytic measles virus into tumors: determinants of efficient loading. Virol Sin 2018;33:234-40.

30 Draganov DD, Santidrian AF, Minev I, et al. Delivery of oncolytic vaccinia virus by matched allogeneic stem cells overcomes critical innate and adaptive immune barriers. J Trans/ Med 2019;17:100-22.

31 Guo Y, Zhang Z, Xu X, et al. Menstrual blood-derived stem cells as delivery vehicles for oncolytic adenovirus virotherapy for colorectal cancer. Stem Cells Dev 2019;28:882-96.

32 Mooney R, Majid AA, Batalla-Covello J, et al. Enhanced delivery of oncolytic adenovirus by neural stem cells for treatment of metastatic ovarian cancer. Mol Ther Oncolytics 2019;12:79-92.

33 Martinez-Quintanilla J, He D, Wakimoto H, et al. Encapsulated stem cells loaded with hyaluronidase-expressing oncolytic virus for brain tumor therapy. Mol Ther 2015;23:108-18.

34 Melen GJ, Franco-Luzón L, Ruano D, et al. Influence of carrier cells on the clinical outcome of children with neuroblastoma treated with high dose of oncolytic adenovirus delivered in mesenchymal stem cells. Cancer Lett 2016;371:161-70.

35 Ruano D, López-Martín JA, Moreno L, et al. First-in-human, first-in-child trial of autologous MscS carrying the oncolytic virus lcovir-5 in patients with advanced tumors. Mol Ther 2020;28:1033-42.

36 VanSeggelen H, Tantalo DG, Afsahi A, et al. Chimeric antigen receptor-engineered T cells as oncolytic virus carriers. Mol Ther Oncolytics 2015;2:15014.

37 Bradley RR, Maxfield LF, Lynch DM, et al. Adenovirus serotype 5-specific neutralizing antibodies target multiple hexon hypervariable regions. J Virol 2012;86:1267-72.

38 Roberts DM, Nanda A, Havenga MJE, et al. Hexon-chimaeric adenovirus serotype 5 vectors circumvent pre-existing anti-vector immunity. Nature 2006;441:239-43.

39 Kuhn I, Harden P, Bauzon M, et al. Directed evolution generates a novel oncolytic virus for the treatment of colon cancer. PLoS One 2008;3:1-11.

40 Thirunavukarasu P, Sathaiah M, Gorry MC, et al. A rationally designed A34R mutant oncolytic poxvirus: improved efficacy in peritoneal carcinomatosis. Mol Ther 2013;21:1024-33.

41 Rojas JJ, Sampath P, Bonilla B, et al. Manipulating TLR signaling increases the anti-tumor $T$ cell response induced by viral cancer therapies. Cell Rep 2016;15:264-73.

42 Tuzmen C, Cairns TM, Atanasiu D, et al. Point mutations in retargeted gD eliminate the sensitivity of EGFR/EGFRvIII-Targeted HSV to key neutralizing antibodies. Mol Ther Methods Clin Dev 2020;16:145-54.

43 Ferguson MS, Lemoine NR, Wang Y. Systemic delivery of oncolytic viruses: hopes and hurdles. Adv Virol 2012;2012:805629. 
44 Adair RA, Roulstone V, Scott KJ, et al. Cell carriage, delivery, and selective replication of an oncolytic virus in tumor in patients. Sci Transl Med 2012;4:138ra77 LP-138ra77.

45 Niemann J, Woller N, Brooks J, et al. Molecular retargeting of antibodies converts immune defense against oncolytic viruses into cancer immunotherapy. Nat Commun 2019;10:3236.

46 Bian H, Fournier P, Moormann R, et al. Selective gene transfer in vitro to tumor cells via recombinant Newcastle disease virus. Cancer Gene Ther 2005;12:295-303.

47 Bian H, Fournier P, Peeters B, et al. Tumor-targeted gene transfer in vivo via recombinant Newcastle disease virus modified by a bispecific fusion protein. Int J Oncol 2005;27:377-84.

48 Slaney CY, Wang P, Darcy PK, et al. Cars versus bites: a comparison between $\mathrm{T}$ Cell-Redirection strategies for cancer treatment. Cancer Discov 2018;8:924-34.

49 Ricca JM, Oseledchyk A, Walther T, et al. Pre-existing immunity to oncolytic virus potentiates its immunotherapeutic efficacy. Mol Ther 2018;26:1008-19.

50 Matveeva OV, Chumakov PM. Defects in interferon pathways as potential biomarkers of sensitivity to oncolytic viruses. Rev Med Virol 2018;28:1-13.

51 Hou F, Sun L, Zheng $\mathrm{H}$, et al. Mavs forms functional prion-like aggregates to activate and propagate antiviral innate immune response. Cell 2011;146:448-61.

52 Pak-Wittel MA, Yang L, Sojka DK, et al. Interferon- $\gamma$ mediates chemokine-dependent recruitment of natural killer cells during viral infection. Proc Natl Acad Sci U S A 2013;110:E50-9.

53 Ying $\mathrm{L}$, Cheng $\mathrm{H}$, Xiong XW, et al. Interferon alpha antagonizes the anti-hepatoma activity of the oncolytic virus $\mathrm{M} 1$ by stimulating antiviral immunity. Oncotarget 2017;8:24694-705.

54 Stewart CE, Randall RE, Adamson CS. Inhibitors of the interferon response enhance virus replication in vitro. PLoS One 2014;9:3-10

55 Ghonime MG, Cassady KA. Combination therapy using ruxolitinib and oncolytic HSV renders resistant MPNSTs susceptible to virotherapy. Cancer Immunol Res 2018;6:1499-510.

56 Patel MR, Jacobson BA, Ji Y, et al. Vesicular stomatitis virus expressing interferon- $\beta$ is oncolytic and promotes antitumor immune responses in a syngeneic murine model of non-small cell lung cancer. Oncotarget 2015;6:33165-77.

57 Phan M, Watson MF, Alain T, et al. Oncolytic viruses on drugs: achieving higher therapeutic efficacy. ACS Infect Dis 2018;4:1448-67.

58 Le Bœuf F, Batenchuk C, Vähä-Koskela M, et al. Model-based rational design of an oncolytic virus with improved therapeutic potential. Nat Commun 2013;4:1974.

59 Sarén T, Ramachandran M, Martikainen M, et al. Insertion of the type-I IFN decoy receptor B18R in a miRNA-Tagged Semliki Forest virus improves oncolytic capacity but results in neurotoxicity. Mol Ther Oncolytics 2017;7:67-75.

60 Selman M, Rousso C, Bergeron A, et al. Multi-modal potentiation of oncolytic virotherapy by vanadium compounds. Mol Ther 2018;26:56-69.

61 Selman M, Ou P, Rousso C, et al. Dimethyl fumarate potentiates oncolytic virotherapy through NF-кB inhibition. Sci Trans/ Med 2018;10:eaao1613. doi:10.1126/scitransImed.aao1613

62 Alvarez-Breckenridge CA, Yu J, Kaur B, et al. Deciphering the multifaceted relationship between oncolytic viruses and natural killer cells. Adv Virol 2012;2012:702839.

63 Li F, Sheng Y, Hou W, et al. CCL5-armed oncolytic virus augments CCR5-engineered NK cell infiltration and antitumor efficiency. $J$ Immunother Cancer 2020;8:1-12.

64 Yoo JY, Jaime-Ramirez AC, Bolyard C, et al. Bortezomib treatment sensitizes oncolytic HSV-1-treated tumors to NK cell immunotherapy. Clin Cancer Res 2016;22:5265-76.

65 Alkayyal AA, Tai L-H, Kennedy MA, et al. Nk-Cell recruitment is necessary for eradication of peritoneal carcinomatosis with an IL12-expressing Maraba virus cellular vaccine. Cancer Immunol Res 2017;5:211-21.

66 Jennings VA, Scott GB, Rose AMS, et al. Potentiating oncolytic virus-induced immune-mediated tumor cell killing using histone deacetylase inhibition. Mol Ther 2019;27:1139-52.

67 Alvarez-Breckenridge CA, Yu J, Price R, et al. Nk cells impede glioblastoma virotherapy through NKp30 and NKp46 natural cytotoxicity receptors. Nat Med 2012;18:1827-34.

68 Xu B, Ma R, Russell L, et al. Amendments: publisher correction: an oncolytic herpesvirus expressing E-cadherin improves survival in mouse models of glioblastoma. Nat Biotechnol 2019;37:45-62. doi:10.1038/nbt0119-102c

69 Altomonte J, Wu L, Meseck M, et al. Enhanced oncolytic potency of vesicular stomatitis virus through vector-mediated inhibition of NK and NKT cells. Cancer Gene Ther 2009;16:266-78.
$70 \mathrm{Kim}$ Y, Yoo JY, Lee TJ, et al. Complex role of NK cells in regulation of oncolytic virus-bortezomib therapy. Proc Natl Acad Sci U S A 2018;115:4927-32.

71 Chen X, Han J, Chu J, et al. A combinational therapy of EGFR-CAR NK cells and oncolytic herpes simplex virus 1 for breast cancer brain metastases. Oncotarget 2016;7:27764-77.

72 Pourchet A, Fuhrmann SR, Pilones KA, et al. CD8(+) T-cell Immune Evasion Enables Oncolytic Virus Immunotherapy. EBioMedicine 2016;5:59-67.

73 Kim M, Nitschké M, Sennino B, et al. Amplification of oncolytic vaccinia virus widespread tumor cell killing by sunitinib through multiple mechanisms. Cancer Res 2018;78:922-37.

74 Jiang H, Rivera-Molina Y, Gomez-Manzano C, et al. Oncolytic adenovirus and tumor-targeting immune modulatory therapy improve autologous cancer vaccination. Cancer Res 2017;77:3894-907.

75 Rivera-Molina Y, Jiang H, Fueyo J, et al. GITRL-armed Delta-24RGD oncolytic adenovirus prolongs survival and induces antiglioma immune memory. Neurooncol Adv 2019;1:1-11.

76 Nakao S, Arai Y, Tasaki M, et al. Intratumoral expression of IL-7 and IL-12 using an oncolytic virus increases systemic sensitivity to immune checkpoint blockade. Sci Trans/ Med 2020;12. doi:10.1126/scitranslmed.aax7992. [Epub ahead of print: 15 Jan 2020].

77 Sivanandam V, LaRocca CJ, Chen NG, et al. Oncolytic viruses and immune checkpoint inhibition: the best of both worlds. Mol Ther Oncolytics 2019;13:93-106.

78 Ribas A, Dummer R, Puzanov I, et al. Oncolytic virotherapy promotes intratumoral T cell infiltration and improves anti-PD-1 immunotherapy. Cell 2017;170:1109-19.

79 Aiken R, Chen C, Cloughesy T, et al. ATIM-33. interim results of a phase II multi-center study of oncolytic adenovirus DNX-2401 with pembrolizumab for recurrent glioblastoma; captive study (KEYNOTE-192). Neuro Oncol 2019;21:vi8-9.

80 Rodríguez-García A, Svensson E, Gil-Hoyos R, et al. Insertion of exogenous epitopes in the E3-19K of oncolytic adenoviruses to enhance TAP-independent presentation and immunogenicity. Gene Ther 2015;22:596-601.

81 McGray AJR, Huang R-Y, Battaglia S, et al. Oncolytic Maraba virus armed with tumor antigen boosts vaccine priming and reveals diverse therapeutic response patterns when combined with checkpoint blockade in ovarian cancer. J Immunother Cancer 2019;7:1-16.

82 Ozcan G, Ozpolat B, Coleman RL, et al. Preclinical and clinical development of siRNA-based therapeutics. Adv Drug Deliv Rev 2015;87:108-19.

83 Tekedereli I, Alpay SN, Tavares CDJ, et al. Targeted silencing of elongation factor 2 kinase suppresses growth and sensitizes tumors to doxorubicin in an orthotopic model of breast cancer. PLoS One 2012;7:e41171.

84 Hamurcu Z, Ashour A, Kahraman N, et al. Foxm1 regulates expression of eukaryotic elongation factor 2 kinase and promotes proliferation, invasion and tumorgenesis of human triple negative breast cancer cells. Oncotarget 2016;7:16619-35.

85 Bayraktar R, Ivan C, Bayraktar E, et al. Dual suppressive effect of miR-34a on the FOXM1/EEF2-kinase axis regulates triplenegative breast cancer growth and invasion. Clin Cancer Res 2018;24:4225-41.

86 Rothlin CV, Ghosh S, Zuniga El, et al. Tam receptors are pleiotropic inhibitors of the innate immune response. Cell 2007;131:1124-36.

87 Pearson RM, Casey LM, Hughes KR, et al. In vivo reprogramming of immune cells: technologies for induction of antigen-specific tolerance. Adv Drug Deliv Rev 2017;114:240-55.

88 Otsuka M, Tsuchiya S, Aramaki Y. Involvement of ERK, a MAP kinase, in the production of TGF-beta by macrophages treated with liposomes composed of phosphatidylserine. Biochem Biophys Res Commun 2004;324:1400-5.

89 Allavena P, Sica A, Garlanda C, et al. The yin-yang of tumorassociated macrophages in neoplastic progression and immune surveillance. Immunol Rev 2008;222:155-61.

90 Pang L, Macauley MS, Arlian BM, et al. Encapsulating an immunosuppressant enhances tolerance induction by SiglecEngaging tolerogenic liposomes. Chembiochem 2017;18:1226-33.

91 Rodriguez-Fernandez S, Pujol-Autonell I, Brianso F, et al. Phosphatidylserine-liposomes promote tolerogenic features on dendritic cells in human type 1 diabetes by apoptotic mimicry. Front Immunol 2018;9:253.

92 Getts DR, Martin AJ, McCarthy DP, Terry RL, Hunter ZN, et al. Microparticles bearing encephalitogenic peptides induce T-cell tolerance and ameliorate experimental autoimmune encephalomyelitis. Nat Biotechnol 2012;30:1217-24. 
93 Cappellano G, Woldetsadik AD, Orilieri E, et al. Subcutaneous inverse vaccination with PLGA particles loaded with a MOG peptide and IL-10 decreases the severity of experimental autoimmune encephalomyelitis. Vaccine 2014;32:5681-9.

94 Yeste A, Nadeau M, Burns EJ, et al. Nanoparticle-mediated codelivery of myelin antigen and a tolerogenic small molecule suppresses experimental autoimmune encephalomyelitis. Proc Nat/ Acad Sci U S A 2012;109:11270-5.

95 Meliani A, Boisgerault F, Hardet R, et al. Antigen-selective modulation of $\mathrm{AAV}$ immunogenicity with tolerogenic rapamycin nanoparticles enables successful vector re-administration. Nat Commun 2018:9:4098.

96 Flórez-Grau G, Zubizarreta I, Cabezón R, et al. Tolerogenic dendritic cells as a promising antigen-specific therapy in the treatment of multiple sclerosis and neuromyelitis optica from preclinical to clinical trials. Front Immunol 2018:9:1-10.

97 Tran TTP, Eichholz K, Amelio P, et al. Humoral immune response to adenovirus induce tolerogenic bystander dendritic cells that promote generation of regulatory T cells. PLOS Pathog 2018;14:e1007127.

98 Sobhanimonfared F, Bamdad T, Sadigh ZA, et al. Virus specific tolerance enhanced efficacy of cancer immuno-virotherapy. Microb Pathog 2020;140:103957.

99 Guedan S, Alemany R. Car-T cells and oncolytic viruses: joining forces to overcome the solid tumor challenge. Front Immunol 2018;9:2460.

100 Nishio N, Diaconu I, Liu H, et al. Armed oncolytic virus enhances immune functions of chimeric antigen receptor-modified T cells in solid tumors. Cancer Res 2014;74:5195-205. 